\title{
Relación entre calidad de vida y representación de enfermedad en personas con enfermedad renal crónica terminal en tratamiento con hemodiálisis
}

\author{
Claudia Pérez Acuña ${ }^{1}$, Giselle Riquelme Hernández², Judith Scharager Goldenberg ${ }^{3}$, Iván Armijo Rodríguez ${ }^{4}$ \\ ${ }^{1}$ Enfermera-Matrona, Magíster en Psicología de la Salud, Directora Carrera de Enfermería, ${ }^{2}$ Matrona, Magíster en \\ Enfermería, Docente Carrera de Enfermería, Facultad de Medicina Clínica Alemana-Universidad del Desarrollo. \\ Chile. ${ }^{3}$ Psicóloga, Magíster en Salud Pública, ${ }^{4}$ Psicólogo, Magíster en Ciencias, Docentes Facultad de Ciencias \\ Sociales, Pontificia Universidad Católica de Chile. Santiago. Chile
}

\section{Resumen}

Introducción: La hemodiálisis produce alteración de las actividades diarias de las personas con enfermedad renal crónica terminal que deterioran significativamente su calidad de vida. La forma en que estas personas representan su enfermedad influye en el control de la patología y en la calidad de vida. Objetivo: evaluar la asociación entre representación de enfermedad y percepción de calidad de vida en personas con enfermedad renal crónica terminal en hemodiálisis. Metodología: estudio correlacional predictivo, de corte transversal realizado en 100 personas con enfermedad renal crónica en hemodiálisis. Se utilizaron como instrumentos el IPQ-R (representación de enfermedad) y el KDQOLSF (medición de la calidad de vida). Se aplicaron estadísticos descriptivos, analíticos y análisis de sendero. Resultados: se observó una puntuación más alta en la percepción de calidad de vida específica v/s genérica. En la representación de enfermedad se observa que aquellos que perciben menos signos y síntomas presen$\tan$ menos consecuencias $(r=0.317 ; p=0.001)$, asimismo, quienes identifican menos consecuencias presentan menos síntomas psicológicos $(r=0.496 ; p=0.000)$ y mayor comprensión de la enfermedad $(r=-0.428 ; p=$ 0.000 ). En la relación entre calidad de vida y representación de enfermedad se encontró una correlación inversa entre la percepción de signos y síntomas (identidad) y la percepción de calidad de vida general $(r$ $=-.403 ; p=0.000)$ y específica $(r=-.380 ; p=0.000)$. Discusión: es importante considerar la percepción del usuario en la evaluación de la calidad de vida y la representación de enfermedad, para favorecer su empoderamiento respecto de su enfermedad.

\section{Correspondencia: \\ Claudia Pérez Acuña \\ Avda. La Plaza 680}

San Carlos de Apoquindo, Las Condes. Santiago. Chile

E-mail: claudiaperez@udd.cl
PALABRAS CLAVE

- REPRESENTACIÓN DE ENFERMEDAD

- CALIDAD DE VIDA

- ENFERMEDAD RENAL CRÓNICA TERMINAL

- HEMODIÁLISIS

Relationship between quality of life and representation of disease in people with chronic renal illness

\begin{abstract}
Introduction: Hemodialysis produces an alteration of the daily activities of people with chronic kidney disease that significantly impair their quality of life. How these people represent their condition affects the control of disease and quality of life. Objective: To assess the association between illness representation and perception of quality of life in people with chronic kidney disease on hemodialysis. Methodology: predictive correlational cross-sectional study conducted in 100 patients with chronic kidney disease on hemodialysis. Were used as instruments the IPQ-R (representation of disease) and KDQOLSF (measuring quality of life). We applied descriptive statistical, analytical and path analysis. Results: A higher score on the perception of specific quality of life was observed vs generic. In the representation of disease is observed that those who earn less signs and symptoms have fewer consequences $(r=0.317$; $p=0.001$ ), also, who identify less consequences have fewer psychological symptoms $(r=0.496 ; p=$ $0.000)$ and greater understanding of the disease $(r=$ $-0,428 ; p=0.000$ ). The relationship between quality of life and disease representing an inverse correlation between the perception of signs and symptoms (identity) and the perceived overall quality of life was found $(r=-403 ; . P=0.000)$ and specific $(r=-.380$;
\end{abstract}


$p=0.000$ ). Discussion: It is important to consider the user's perception in evaluating the quality of life and the representation of disease, to promote their empowerment regarding their disease.

\section{KEYWORDS}

- REPRESENTATION OF DISEASE

- QUALITY OF LIFE

- CHRONIC RENAL FAILURE

- HEMODIALYSIS

\section{Introducción}

La enfermedad renal crónica terminal (ERCT) se ha constituido en los últimos años en un problema de salud pública que requiere tratamientos de sustitución renal por diálisis o trasplante renal ${ }^{1}$. La hemodiálisis (HD) produce una alteración de las actividades diarias de la persona con ERCT, al provocar cambios físicos, psicológicos, sociales, familiares, económicos y laborales que deterioran significativamente su calidad de vida ${ }^{2-4}$.

La calidad de vida relacionada con la salud (CVRS), definida como la percepción que tiene el sujeto de los efectos de una enfermedad determinada o de la aplicación de cierto tratamiento en diversos ámbitos de su vida se encuentra influenciada por distintos factores ${ }^{5-7}$. En personas en HD se ha observado que la CVRS se ve influenciada por factores sociodemográficos (edad, género, nivel socioeconómico, nivel educacional y situación laboral $)^{4,7-15}$; factores psicosociales (apoyo social y familiar, ansiedad, depresión)4,7,13-15,17-21; y factores clínicos (comorbilidad, síntomas físicos, nivel de hematocrito-hemoglobina) $2,7,11,13,15,20,22-24$. Distintos estudios muestran un deterioro en todas las dimensiones de la CVRS de las personas en HD, siendo la más afectada la dimensión física ${ }^{7,8,14,20,23,25,26}$.

Considerando los factores que influyen, la CVRS dependería en que los mecanismos de afrontamiento y la adecuación de las expectativas de la persona enferma, permitieran que la brecha entre expectativa y percepción de la situación actual no fuese tan desproporcionada 6 .

La visión de una enfermedad crónica es dinámica y está basada en la interpretación, evaluación y percepción de resultados de las estrategias utilizadas para enfrentarla, formando una representación cognitiva de la enfermedad que influye en su mejor o peor adaptación a la misma ${ }^{27,28}$. El modelo de autorregulación de Leventhal muestra una conducta de enfermedad en la cual la persona es concebida como un ente activo que desarrolla sus propias teorías en torno a su estado de salud. Estas teorías tienen un gran valor, pues en ellas quedarán recogidos los factores que la persona enferma juzga importantes, como la causa de la enfermedad, su evolución y duración ${ }^{29,30}$. Según este modelo, la representación de enfermedad posee cinco atributos: identidad de la enfermedad, consecuencias, causas, temporalidad o curso, y controlabilidad; de la combinación de los factores anteriores se obtienen tres diferentes esquemas de enfermedad: agudo, crónico y cíclico ${ }^{30}$.

Las investigaciones en esta área están centradas en los factores clínicos biológicos que afectan a la persona con ERCT, sin considerar cómo estos pacientes representan su enfermedad $2,4,7,11,13,15$. Estas representaciones pueden afectar las conductas que las personas adoptan en el manejo de su enfermedad y la regulación de la actividad diaria, lo que se asocia con una menor percepción de la CVRS $^{31}$. El significado que la persona en hemodiálisis da a su enfermedad puede influenciar sus respuestas para hacer frente a su nivel de funcionalidad física ${ }^{31}$.

Estudios en ERCT basados en el modelo de autorregulación y su relación con la CVRS, mostraron que a más manifestaciones emocionales y consecuencias percibidas, menor era la percepción de CVRS, asimismo, quienes presentan más sintomatología lo asocian con mayores consecuencias y sintomatología psicológica, y aquellas que representan su enfermedad cómo crónica, perciben mayores consecuencias y tienen menor control personal para manejar su enfermedad ${ }^{32-34}$.

Al reconocer la representación de enfermedad como un factor que influye en el control de la patología y en la CVRS, se podrán plantear intervenciones específicas que permitan una mejor adaptación de la persona frente a su enfermedad y tratamiento, aumentando la percepción de la CVRS ${ }^{30}$. En este contexto, el objetivo de esta investigación fue evaluar la asociación entre representación de enfermedad y percepción de CVRS en personas con ERCT en HD. 


\section{Material y métodos}

Estudio correlacional predictivo, de corte transversal, con muestra no probabilística, intencional de 100 personas bajo los siguientes criterios de inclusión: a) hombres y mujeres mayores de 20 años, b) alfabetos y analfabetos, c) diagnosticados con ERCT, d) tratamiento en HD definitiva mayor a un año, e) sin dificultad comunicacional.

Para la recolección de datos se aplicaron tres instrumentos:

1) Encuesta datos sociodemográficos y clínicos construida por los investigadores.

2) Escala de medición de representación de enfermedad "IIIness Perception Questionnaire-Revised $(I P Q-R)^{\prime \prime}$, adaptado en personas con ERCT en HD por Thomas-Hawkins (2004) conformada por tres secciones:

a) presencia/ausencia de 11 síntomas (subescala de identidad).

b) 38 ítems referidos a las dimensiones de control personal, control del tratamiento, duración (aguda/crónica), curso (cíclico), consecuencias, representaciones emocionales y comprensión de la enfermedad.

c) 18 opciones de las posibles causas de la enfermedad. Para su utilización, el IPQ-R fue traducido y aplicado en dos pruebas pilotos, modificándose 7 preguntas de la sección 2 , sin modificaciones en las secciones 1 y 3 .

3) Escala para medir la CVRS "Kidney Disease Quality of Life- short Form" (KDQOL-SF), versión en español ${ }^{35}$, compuesta por dos partes: una genérica de 36 ítems que mide ocho dimensiones del estado de salud (función física, rol físico, dolor, salud general, vitalidad, función social, rol emocional y salud mental); y una específica para personas en HD, que incluye 43 ítems específicos de la enfermedad renal que conforman un total de 11 escalas (sintomatología, efecto de la enfermedad renal, carga de la enfermedad renal, trabajo, función cognitiva, calidad de la interacción social, función sexual, sueño, apoyo social, apoyo del personal de diálisis y satisfacción del paciente ${ }^{13}$. Para su aplicación, previa autorización del autor, se realizó prueba piloto para evaluar la comprensión del contenido y lenguaje. No se realizaron modificaciones al instrumento original.

La recolección de datos se hizo durante la sesión de HD, previa firma de un consentimiento informado.
Los datos fueron analizados con el programa SPSS. Para el estudio del KDQOL-SF se aplicó la prueba $T$ para muestras relacionadas y coeficiente alfa de Cronbach para determinar la consistencia interna de las subescalas. En el instrumento IPQ$R$ las intercorrelaciones entre las subescalas se calcularon mediante coeficiente de Pearson. En el análisis de asociación de las subescalas de representación de enfermedad y CVRS, se aplicó el coeficiente de Pearson para luego realizar un análisis de sendero, con el fin de predecir la percepción de la CVRS.

\section{Resultados}

La características sociodemográficas y el perfil médico-clínico de los participantes se muestran en la tabla I.

Tabla 1. Características sociodemográficas y médico-clínicas de los pacientes del estudio.

\begin{tabular}{|c|c|c|}
\hline $\begin{array}{c}\text { Características sociodemográficas } \\
\text { y médico-clínicas }\end{array}$ & Promedio & DS \\
\hline \multicolumn{3}{|l|}{ Género } \\
\hline Hombres & 53 & - \\
\hline Mujeres & 47 & - \\
\hline Edad (años) & 56,3 & 16,01 \\
\hline \multicolumn{3}{|l|}{ Nivel máximo de estudios realizados } \\
\hline Educación básica & 14 & - \\
\hline Educación media & 35 & - \\
\hline Educación técnica & 15 & - \\
\hline Ediucación universitaria & 36 & - \\
\hline \multicolumn{3}{|l|}{ Situación laboral } \\
\hline Activa & 53 & - \\
\hline No activa & 47 & - \\
\hline \multicolumn{3}{|l|}{ Estado civil } \\
\hline Casado & 68 & - \\
\hline Soltero & 19 & - \\
\hline Viudo & 10 & - \\
\hline Separado & 3 & - \\
\hline $\begin{array}{c}\text { Meses en HD } \\
\text { (media + desviación estándar) }\end{array}$ & 64,37 & 57,25 \\
\hline \multicolumn{3}{|l|}{ Comorbilidad } \\
\hline Hipertensión arterial & 64 & - \\
\hline $\begin{array}{l}\text { Diabetes Mellitus + } \\
\text { hipertensión arterial }\end{array}$ & 21 & - \\
\hline Diabetes Mellitus & 1 & - \\
\hline Otras patologías & 14 & - \\
\hline$\%$ Hematocrito & 29,38 & 5,27 \\
\hline \multicolumn{3}{|l|}{ Administración de eritropoyetina } \\
\hline Si & 60 & - \\
\hline No & 40 & - \\
\hline
\end{tabular}


En la descripción de CVRS, todos los dominios de la dimensión genérica, excepto salud general $(44,36$ puntos) y vitalidad (58 puntos), presentaron puntajes superiores a 60 puntos. Los dominios de salud mental y rol emocional obtuvieron los mayores puntajes $(69,72$ y 69,33 respectivamente). La percepción de la CVRS específica obtuvo valores más altos que la genérica (70 puntos). Los dominios de carga producida por la enfermedad renal $(48,00)$ y condición laboral $(49,50)$ obtuvieron los puntajes más bajos, mientras que los dominios función cognitiva $(82,86)$ e interacción social $(81,33)$, obtuvieron los puntajes más altos.

En la tabla II se muestran los resultados del cuestionario IPQ-R de representación de enfermedad.

Tabla 2. Resultados de las diferentes escalas que se recogen en el cuestionario IPQ-R.

\begin{tabular}{lccc}
\hline Escala & Rango & Media & DS \\
\hline Identidad (11) & $0-11$ & 4,31 & 2,75 \\
Temporalidad o curso (aguda-crónico) (6) & $6-30$ & 23,88 & 5,66 \\
Curso (cíclico) (4) & $4-20$ & 11,55 & 4,22 \\
Consecuencias (6) & $6-30$ & 22,7 & 4,42 \\
Control personal (6) & $6-30$ & 22,03 & 3,69 \\
Control del tratamiento (5) & $5-25$ & 17,04 & 2,88 \\
Comprensión de la enfermedad (5) & $5-25$ & 17,44 & 4,62 \\
Representaciones emocionales (6) & $6-30$ & 18,38 & 5,78 \\
\hline
\end{tabular}

La muestra presentó puntajes altos en curso agudo/ crónico, consecuencias y control personal, encontrándose puntajes más bajos en identidad y curso cíclico. Los síntomas más frecuentes fueron sentirse cansado, agotado y $\sin$ fuerza $(63 \%)$ y prurito $(51 \%)$. Las atribuciones de causalidad más reportada fueron la mala suerte (56\%) y poco cuidado médico en el pasado $(55 \%)$. Dentro de las consecuencias el $99 \%$ piensa que la enfermedad renal es seria, $82 \%$ que tiene grandes consecuencias en su vida y $81 \%$ que tiene serias consecuencias económicas. En control personal el $82 \%$ cree que puede hacer mucho para controlar sus síntomas, $74 \%$ considera que sus comportamientos afectarán el curso de su enfermedad renal y el $67 \%$ que la evolución de la enfermedad renal depende de ellos. En la representación emocional, al 73\% les preocupa la enfermedad renal, $66 \%$ se siente asustado por la enfermedad, $60 \%$ se siente enojado, $54 \%$ se siente ansioso y un $52 \%$ se deprime cuando piensa en la enfermedad renal.
En este estudio se observó una puntuación más alta en la percepción de la CVRS específica $(M=69,66)$ que en la genérica $(M=62.64)$ (diferencia estadísticamente significativa $t=4,858 ; g=99 ; p<0,000$ ). También se observaron puntajes más altos en la dimensión psicológica $(M=65,68)$ que la dimensión física $(M=59,17)(t=3,023 ; g l=99 ; p<0,003) ; y$ una puntuación mayor en la dimensión social $(M=67,87)$ que la dimensión física $(M=59,17)(t=3,123 ; g l=$ 99; $p<0,002$ ).

En la representación de enfermedad se observa que aquellos que perciben menos signos y síntomas presentan menos consecuencias $(r=0.317 ; p=0.001)$, asimismo, quienes identifican menos consecuencias presentan menos síntomas psicológicos $(r=0.496$; $p=0.000$ ) y mayor comprensión de la enfermedad $(r=-0.428 ; p=0.000)$. No se encontró relación entre el control del tratamiento y la comprensión de la enfermedad $(r=0.192 ; p=$ 0.056).

En la relación entre CVRS y representación de enfermedad se encontró una correlación inversa entre la percepción de signos y síntomas (identidad) y la CVRS general $(r=-.403$; $p=0.000)$ y específica $(r=-.380 ; p=0.000)$. De igual forma, se observó una correlación inversa entre las consecuencias de ERCT y la percepción de CVRS general $(r=-.512 ; p=$ $0.000)$ y la especíica $(r=-.485 ; p=0.000)$, indicando que las personas con menos consecuencias muestran una mejor percepción de la CVRS genérica y específica.

Con respecto al control personal y control del tratamiento, existe una directa relación entre quienes poseen mayor control de ambas variables con una mejor percepción de la CVRS general y específica $(r=.258 ; p=0.01$ y $r=.288 ; p=0.004 ;(r=.219 ;$ $p=0.028$ y $r=.238 ; p=0.017$, respectivamente). En la representación emocional de la enfermedad v/s CVRS general y específica, se encontró una correlación inversa $(r=-.553 ; p=0.000 ;(r=-.585$; $p=0.000$, respectivamente), observándose que las personas que tienen menos síntomas psicológicos, muestran una mejor percepción de la CVRS general y específica.

En el análisis de sendero, la figura 1 muestra que tanto la identidad como el curso agudo/crónico tienen un efecto directo sobre la CVRS específica (a más síntomas y curso crónico de enfermedad, menor percepción de CVRS). 


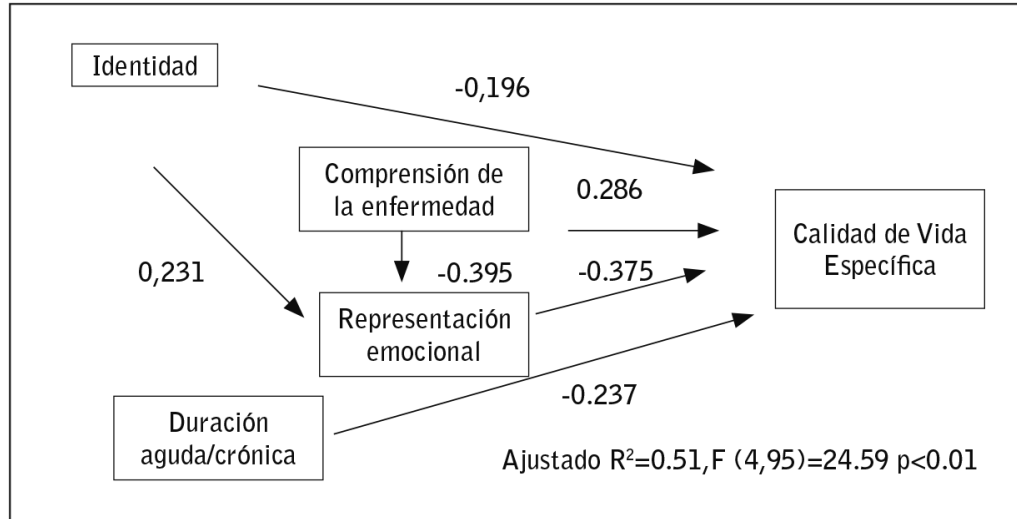

Figura 1. Análisis de sendero que predice la percepción de calidad de vida específica.

tiene una acción indirecta sobre CVRS general. La representación emocional también forma un trayecto directo sobre curso cíclico con un coeficiente muy alto, y curso cíclico a su vez una relación inversa sobre CVRS general, destacando una trayectoria inversa de duración aguda/crónica sobre CVRS general.

\section{Discusión}

La percepción de CVRS general observada en estos participantes es menor que la observada en el ámbito específico, as-

La representación emocional actúa como mediador, influyendo en la relación entre la comprensión de la enfermedad y la CVRS específica. Se identificaron dos senderos: uno que se inicia con el atributo identidad con un efecto positivo sobre el mediador representación emocional y éste a su vez, tiene una asociación inversa sobre CVRS específica. El segundo camino se forma a partir de la comprensión de la enfermedad con un efecto negativo sobre el mediador representación emocional, el cual tiene un efecto negativo sobre la CVRS específica. Cabe señalar también la relación negativa entre curso agudo/crónico y CVRS específica.

En la figura 2 se observa que el curso agudo/crónico e identidad tienen un efecto directo sobre la CVRS general (a más síntomas y esquema crónico de enfermedad, menor calidad de vida).

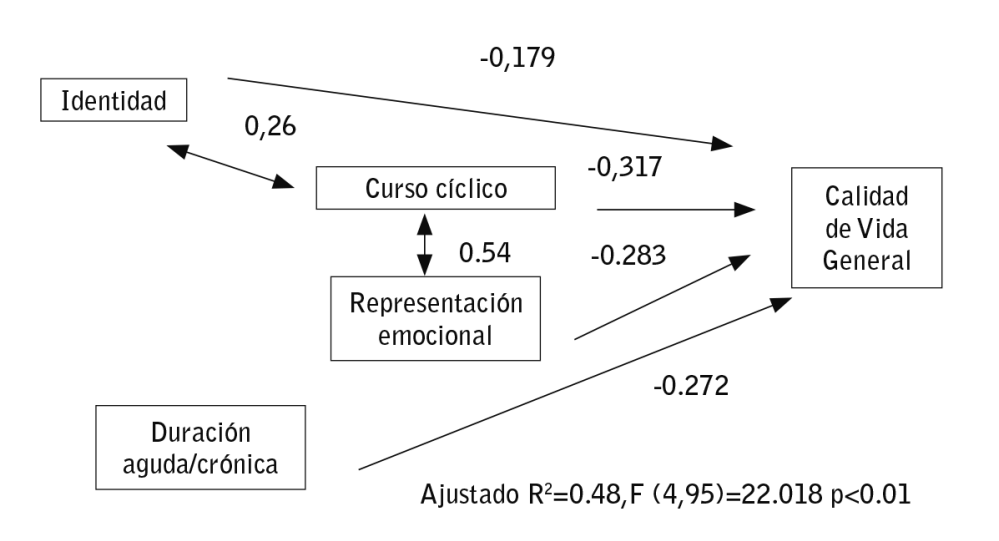

Figura 2. Análisis de sendero que predice la percepción de calidad de vida general. pecto concordante con investigaciones realizadas en estos pacientes ${ }^{7,10,16,23}$, observándose también puntajes bajos en estrés o carga por la enfermedad, condición laboral y repercusiones en la vida diaria $^{7,13}$, lo que estaría relacionado con la condición de discapacidad de la ERCT, pues la progresión de la patología va determinando la manifestación de limitaciones físicas, obstaculizando el desempeño laboral3,8,14-16.

La dimensión física está más afectada que la psicológica y social en la escala genérica ${ }^{7,16,23}$, lo que se debería a que la persona con ERCT vive un proceso de adaptación, ya que a medida que la enfermedad progresa, desarrolla ajustes internos y un proceso de adaptación psicológica que le permiten preservar la satisfacción con la vida, llegando incluso a aumentar la percepción de CVRS en estas dimensiones ${ }^{7}$. Por otra parte, el tratamiento de los síntomas físicos (ej. Uso de eritropoyetina) actuaría tanto sobre la dimensión física como psicosocial, mejorando la percepción de la CVRS ${ }^{24}$.

En la representación de enfermedad, los síntomas y signos descritos son concordantes con lo referido en la literatura ${ }^{16}$, al igual que en las consecuencias, siendo la más afectada negativamente el aspecto económico ${ }^{30}$. En el control personal de su enfermedad, la mayoría de los participantes refieren poder manejarla, aspecto importante a considerar, pues en aquellos sin creencia en un control personal se desarrollaría un estado funcional menos

El curso cíclico de la enfermedad actúa como mediador, influyendo en la relación entre la identidad de la enfermedad y CVRS general. Existe acción directa de identidad sobre el mediador curso cíclico y éste favorable. Lograr un control basado en la internalidad ha demostrado estar asociado a más efectividad para el manejo de la enfermedad ${ }^{30}$. Finalmente, en el tipo de causalidad, se observan factores internos y 
externos, surgiendo el concepto de heredabilidad de la enfermedad ${ }^{30}$.

La mayoría de los participantes de este estudio pertenecen al esquema agudo, resultados diferentes a los reportados en otras investigaciones ${ }^{33}$, donde el mayor grupo pertenecían al esquema crónico. Este hallazgo es importante de considerar, pues la transición de un esquema agudo a uno crónico o su combinación, necesita la re-conceptualización de la representación de enfermedad y puede requerir de intervenciones específicas para lograr cambios en la percepción de cada esquema ${ }^{29}$.

Los atributos de representación de enfermedad encontrados fueron semejantes a otros estudios ${ }^{26,32}$, observándose que a más síntomas, más consecuencias físicas, sociales, económicas o psicológicas, y que a mejor comprensión de la enfermedad, hay menos sintomatología psicológica ${ }^{32,33}$. Asimismo, ante más signos y síntomas de la enfermedad, es menor la percepción de CVRS, y frente a menos consecuencias y mayor control de enfermedad, mejor percepción de CVRS $^{26,32,33}$.

Del análisis de sendero, se deduce que a mayor comprensión de la enfermedad menor representación emocional y mayor CVRS. Si el equipo de salud desea lograr efectos sobre la CVRS, las intervenciones deberían dirigirse sobre el proceso de comprensión de la enfermedad, para mejorar la percepción de CVRS, lo que a la vez actúa disminuyendo la representación emocional. Quienes presentan mayor número de síntomas relacionados con un curso cíclico y mayor representación emocional, presentan menor percepción de la CVRS. Intervenir sobre este grupo es más complejo, pues debe modificarse la percepción de curso cíclico de la enfermedad para disminuir la identidad y representación emocional, y así aumentar la percepción de CVRS, lo que significa trabajar la re-conceptualización de la representación de enfermedad.

Un enfoque biopsicosocial fomenta la participación de los individuos en el ciclo salud-enfermedad, transformándolos en los principales protagonistas de este proceso, para lo cual es importante considerar la percepción del usuario, sus deseos y motivaciones en el proceso de toma de decisiones en salud, así como en la evaluación de la CVRS y la representación de enfermedad, lo que permitiría su empoderamiento y de esta manera una mayor responsabilidad respecto de su enfermedad.
Recibido: 19 mayo 2015

Revisado: 20 mayo 2015

Modificado: 21 mayo 2015

Aceptado: 21 mayo 2015

\section{Bibliografía}

1. Ministerio de Salud de Chile [internet]. Santiago: Departamento de Epidemiología; Encuesta Nacional de Calidad de vida y salud (ENCAVI); 2012 [cited 2014 Diciembre 16). Disponible en: http://epi.minsal. cl/estudios-y-encuestas-poblacionales/encuestaspoblacionales/encuesta-nacional-de-calidad-de-viday-salud-encavi/.

2. Contreras F, Esguerra G, Espinosa JC, Gómez V. Estilos de afrontamiento y calidad de vida en pacientes con insuficiencia renal crónica (IRC) en tratamiento de hemodiálisis. Acta Colombiana de Psicología 2007; 10 (2): 169-179. Disponible en: http://repository. ucatolica.edu.co:8080/handle/10983/1035 [consultado el 16 de diciembre de 2014 ].

3. Acosta P, Chaparro L, Rey C. Calidad de vida $y$ estrategias de afrontamiento en pacientes con insuficiencia renal crónica sometidos a hemodiálisis, diálisis peritoneal 0 trasplante renal. Revista Colombiana de Psicología 2008; (17): 9-26.

4. Sánchez JL, Pelayo R, Ibarguen E, Aja A, Saenz A, Incera $M E$ et al. Factores sociológicos y factores de vida relacionada con la salud en pacientes en hemodiálisis. Rev Soc Esp Enferm Nefrol 2011; 14 (2): 98-104.

5. Seguí A, Amador P, Ramos AB. Calidad de vida en pacientes con insuficiencia renal crónica en tratamiento con diálisis. Rev Soc Esp Enferm Nefrol 2010; 13 (3): 155-160.

6. Urzúa A. Calidad de vida relacionada con la salud: Elementos conceptuales. Rev. Med Chile 2010; 138(3): 358-365.

7. Martín F, Reig A, Sarró F, Ferrer R, Arena D, González F, Gil T. Evaluación de la calidad de vida en pacientes de una unidad de hemodiálisis con el cuestionario Kidney Disease Quality of life- short form (KDQOL-SF). Revista Diálisis y Transplante $2004 ; 25(2), 79-92$. 
8. Arenas $M$, Moreno $E$, Reig A, Millán I, Egea J, Amoedo $\mathrm{M}$ et al. Evaluación de la calidad de vida relacionada con la salud mediante las láminas COOP-WOONCA en una población de hemodiálisis. Nefrología 2004; 24(5): 470-479.

9. Alarcón I, Farías D, Barrios S. Factores que influyen en la percepción de calidad de vida de personas adultas bajo terapias de sustitución renal. Horizonte de Enfermería 2009; 20 (1): 67-80.

10. Chiang C, Peng YS, Chiang S, Yang C, He Y, Hung $\mathrm{K}$ et al. Health-related quality of life of hemodialysis patients in taiwan: a multicenter study. Blood Purif. 2004;22(6):490-8. Disponible en: www.karger.com [consultado el 20 octubre de 2007].

11. Kimmel P, Levy N. Psicología y rehabilitación. En Daugirdas J, Blake T. Manual de diálisis. Madrid, España: Editores; 2003. p.433-434.

12. Morsch CM, Goncalves LF, Barros E. Healthrelated quality of life among haemodialysis patients - relationship with clinical indicators, morbidity and mortality. Journal of Clinical Nursing 2006; 15, 498-504.

13. Seica A, Segall L, Verzan C, Vadura N, Madincea $M$, Rusoi $S$ et al. Factors affecting the quality of life of haemodialysis patients from Romania: a multicentric study. Nephrol Dial Transplant 2009; 24: 626-629.

14. Ginieri-Coccossis M, Theofilou P, Synodinou C, Tomaras V, Soldatos C. Quality of life, mental health and health beliefs in haemodialysis and peritoneal dialysis patients: Investigating differences in early and later years of current treatment. BMC Nephrology 2008; 9 (14): 1-9Disponible en: www.biomedcentral.com/14712369/9/14 [consultado el 16 de diciembre de 2014).

15. Park HC, Yoon $\mathrm{H}$, Son J, Jung ES, Joo KW, Chin $\mathrm{HJ}$ et al. Depression and health related quality of life in maintenance hemodialysis patients. Clinical Nephrology 2010; 73: 374 - 380. Disponible en: www.ncbi.nlm.nih.gov. [consultado el 16 de diciembre de 2014].

16. Contreras F, Espinosa JC, Esguerra G. Calidad de vida, autoeficacia, estrategias de afrontamiento y adhesión al tratamiento en pacientes con insuficiencia renal crónica sometidos a hemodiálisis. Psicología y Salud 2008; 18 (2): 165-179. Disponible en: http://revistas.uv.mx/ index.php/psicysalud/article/view/659/1156. [consultado el 16 de diciembre de 2014].

17. Rodríguez M, Castro M, Merino J. Calidad de vida en pacientes renales hemodializados. Ciencia y Enfermería 2005; 11: 47-57.

18. Perales C, García A, Reyes del Paso G. Predictores psicosociales de la calidad de vida en pacientes con insuficiencia renal crónica en tratamiento de hemodiálisis. Nefrología 2012; 32 (5):62230. Disponible en: www.revistanefrologia.com [consultado el 16 de diciembre de 2014].

19. Vinaccia S, Quiceno J. Calidad de vida relacionada con la salud y enfermedad crónica: estudios colombianos. Psychologia. Avances de la disciplina 2012; 6 (1): 123-136. Disponible en: http:// www.redalyc.org/pdf/2972/297225770004.pdf. [consultado el 16 de diciembre de 2014].

20. Cruz JM, Piera L, Bragg-Gresham JL, Feldman, $H$, Port FK. Resultados del estudio internacional de hemodiálisis DOPPS en Europa y España. Nefrología 2003; 23 (5): 437-443.

21. Contreras F, Esguera G, Espinosa JC, Gutiérrez C, Fajardo L. Calidad de vida y adhesión al tratamiento en pacientes con insuficiencia renal crónica en tratamiento de hemodiálisis. Univ Psychol. Bogotá 2006; 5 (3): 487-499. Disponible en: www.scielo. org [consultado el 20 octubre de 2007].

22. Millán R, Gómez C, Gil F, Oviedo G, Villegas F. Calidad de vida relacionada con la salud $y$ prevalencia de síntomas depresivos y ansiosos en pacientes en hemodiálisis, en seis unidades renales de Bogotá, Colombia. Rev Colomb Psiquiat 2009; 38 (4): 622-636.

23. Sociedad Chilena de Nefrología. Registro de diálisis. (2013). Disponible en: http://www.fmcag.cl/ffile/file_2_cuentahemodialisis2013.pdf [consultado el 5 de diciembre de 2014].

24. De Castro M, Santesso A, Draibe $S$, Fernández M. Qualidade de vida de pacientes com insuficiência renal crônica em hemodiálise avaliada a través do instrumento genérico SF-36. Associaçao Médica Brasileira 2003; 49: 245-249.

25. Gregory N. Effect of higher hemoglobin levels on health- related quality of life parameters. Nephrology Nursing Journal 2003; 30(1), 75-78.

26. Pérez C, Scharager J, Armijo I. Percepción de calidad de vida en personas sometidas a hemodiálisis crónica. Horizonte de Enfermería 2007; 18 (2): 21-32. 
27. Santos P, Franco L. Clinical and laboratory variables associated with quality of life in Brazilian haemodialysis patients: a single-centre study. Rev Méd Chile 2008; 136 (10): 12641271.

28. Carlisle A, Jonh, A, Fife-Schaw C, Lloyd M. The self-regulatory model in women with rheumatoid arthritis: Relationships between illness representations, coping strategies, and illness outcome. British Journal of Health Psychology 2005, 10, 571-587. Disponible en: www. cirrie. buffalo.edu/database/28228/. [consultado el 11 marzo de 2007].

29. Leventhal $H$, Lambert J.F, Diefenbach $M$, Leventhal E.A. From compliance to social-selfregulation: Models of the compliance process. In B: Blackwell(Ed), Treatment compliance and the therapeutic alliance. Chronic mental illness Singapore: Harwood Academic Publishers, 2003. p 17-33.

30. Leventhal H, Halm E, Horowitz C, Leventhal EA, Ozakinci G. Living whit chronic Illness:A contextualizad, self-regulation approach. In S.Sutton, M Johnston \& A. Baum (Eds) The sage handbook of health psychology. London: Sage, 2004. p. 197-240.
31. Thomas-Hawkins C. Illness representations and activity self-regulation in elders with ESRD. Nephrology Nursing Journal 2004; 31: 139.

32. Hagger M, Orbell S. A meta-analytic review of the common-sense model of illness representations. Psychology and Health 2003; 18 (2): 141-184.

33. Fowler C, Baas L. Illness representations in patients with chronic kidney disease on maintenance hemodialysis. Nephrology Nursing Journal 2006; 33 (2): 173-174, 179-186.

34. Covic A, Seica A, Gusbeth-Tatomir P, Gavrilovici 0 , Goldsmith DJA. Illness representations and quality of life scores in haemodialysis patients. Nephology Dialysis Transplantation 2004; 19 (8): 2078-2083.

35. García F, López K, De Álvaro F, Álvarez-U de $F$, Alonso J. Salud percibida en pacientes que comienzan tratamiento renal sustitutivo: validación preliminar de la versión española del KDQOL-SF. Nefrología 1998; 18 (3): 66. 